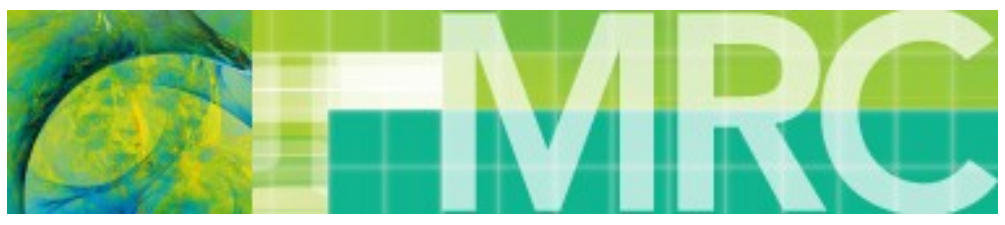

\title{
An Evaluation of Kefir Grain Size with Magnetic Resonance Imaging to Observe the Fermentation of Milk
}

\begin{tabular}{|r|l|}
\hline Journal: & Magnetic Resonance in Chemistry \\
\hline Manuscript ID & MRC-18-0131.R1 \\
\hline Wiley - Manuscript type: & Special Issue Research Article \\
\hline Complete List of Authors: & $\begin{array}{l}\text { Spicer, Abi; Nottingham Trent University - Clifton Campus, Physics and } \\
\text { Mathematics } \\
\text { Fairhurst, David; Nottingham Trent University - Clifton Campus, Physics } \\
\text { and Mathematics } \\
\text { Newton, Michael; Nottingham Trent University - Clifton Campus, Physics } \\
\text { and Mathematics } \\
\text { Morris, Robert; Nottingham Trent University - Clifton Campus, Physics } \\
\text { and Mathematics }\end{array}$ \\
\hline Keywords: & Magnetic Resonance Imaging, Kefir, Fermentation, Milk, Grain Size \\
\hline &
\end{tabular}

\section{SCHOLARONE" \\ Manuscripts}




\title{
An Evaluation of Kefir Grain Size with Magnetic Resonance Imaging to Observe the Fermentation of Milk
}

\author{
Abi Spicer $^{1}$ | David J. Fairhurst ${ }^{1}$ | Michael I. Newton ${ }^{1}$ \\ | Robert H. Morris ${ }^{1 *}$
}

${ }^{1}$ School of Science and Technology, Nottingham Trent University, Clifton Lane, Nottingham, NG11 8NS, UK

\section{Correspondence}

Robert H. Morris, School of Science and Technology, Nottingham Trent University, Clifton, Nottinghamshire, NG11 8NS, UK Email: rob.morris@ntu.ac.uk

Funding information

This work has been internally funded by Nottingham Trent University.

\footnotetext{
${ }^{*}$ Corresponding Author
}

Kefirian milk is a fermented beverage consumed worldwide. Originally produced in animal skins, it is now prepared both industrially and at home by adding symbiotic cultures of bacteria and yeast known as kefir grains to fresh milk. There is significant literature on the biological aspects of this process but little focus on the fermentation effects on the bulk milk as a function of the grain morphology. Changes in the MR signal as measured using a whole body clinical MRI scanner are found to be proportional to traditional gas measurements with correlation coefficients in excess of 0.95. Magnetic Resonance Imaging is then also used to determine the effect of grain size on the rate of fermentation of milk. It is found that larger grains result in signal intensity changes on the order of 0.03 a.u per hour but by breaking the grains into pieces around $3 \mathrm{~mm}$ the reaction rate can be more than doubled to 0.07 a.u. per hour. It is thought that this shows promise as a method to improve the speed of production of kefirian milk and by arresting the process partway through fermentation, gives control over the properties of the end product.

KEYWORDS

Magnetic Resonance Imaging, Kefir, Fermentation, Milk, Grain Size 


\section{1 | INTRODUCTION}

Kefir is a fermented milk beverage that originated in the Caucasus mountains [1]. Originally produced by fermenting milk in animal hide bags, it is now produced by adding symbiotic cultures of bacteria and yeasts, known as kefir grains, to fresh milk. The milk and grain mixture is left overnight at room temperature $\left(20-25^{\circ} \mathrm{C}\right)$ in order to allow the fermentation process to occur [2]. Once fermented, the mixture is strained and the grains are recovered to allow for re-use. The strained liquid, known as kefirian milk, is a lightly carbonated, slightly acidic drink with a thin yogurt-like consistency [2]. Kefirian milk has become a widely consumed beverage due to its known pro-biotic properties and has been claimed to have health benefits such as lowering cholesterol [3]. Commercial production of kefir is challenging owing to the variability in the kefir grains. As a result milk is typically inoculated with the most dominant bacterial strains to give a beverage with similar properties [4] although the complexities of traditional kefir are not well replicated by this approach. Much of the home production of kefir is undertaken by word of mouth guidance and as such, whilst there are attempts to standardize production [5], there is little in the way of 'best practice' to allow for optimization of the method, improving the possibility of developing a process which could be sufficiently quality controlled for commercial purposes.

The flocculation process [6] which drives the increase in viscosity in response to fermentation should provide a change in magnetic resonance relaxation parameters since the agglomeration of milk proteins will restrict the diffusion of bulk water. In this study, the dependence of magnetic resonance signals on fermentation using Magnetic Resonance Imaging (MRI) is first verified before investigating the effect of grain size and milk to volume ratio on the fermentation rate and capability.

\section{1 | Kefir Grain Anatomy}

The kefir grains are a symbiotic colony of bacteria and yeast which are not evenly distributed throughout the structure $[7,8]$. There is some disagreement in the literature [3,9] about the exact nature of the biological makeup of the grains with some suggesting bacteria such as lactobacilli and lactococci are found only on the outer layer of the grain, whilst different yeast strains live within the extracellular matrix that makes up the structure of the grain [7]. For this reason it is thought that the main contribution to fermentation is from the bacterial outer layer which is in direct contact with the milk. During the fermentation process the physical size of the grain increases due to multiplication of the living elements of the colony. After several fermentations, new grains start to appear, formed from the accumulation of the biological components which themselves then increase in size with subsequent fermentation cycles. The mature grains are off-white in colour and resemble cauliflower florets in structure (see Figure 1), ranging in size from a few millimeters to five centimeters $[10,8,11]$. The evolution of the structure of the grains causes a significant increase in surface area with size.

Due to the kefir grains' constant growth, repeated fermentation leads to a production of an excess of grains, which if not removed cause the kefirian milk to taste excessively sour. Home producers often discard these grains, or share them with others. The primary driver of kefir production is to generate the greatest volume in the shortest amount of time whilst retaining the beverage's unique properties. It has been reported in the literature [2] that the milk to grain ratio affects fermentation and so it is likely that the grain size will have an important role in this process. Small grains are often lost during the straining process but can be created by pulling apart large grains, although this somewhat exposes the surface area of the yeast-containing extracellular matrix to the milk. 


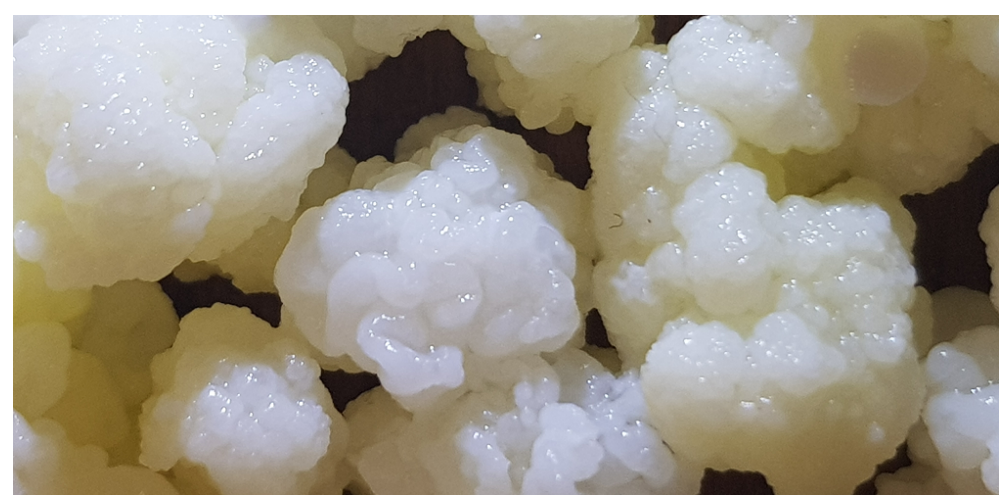

FIGURE 1 A photograph showing the normal morphology of several kefir grains.

\section{2 | Fermentation Measurements}

Kefir has been compared to bakers yeast [12] in its ability to produce visible bubbles of gas during fermentation. As with any such fermentation process, a viable method to measure the fermentation rate is to collect the gas produced as a function of time [13]. Gas measurements however have limitations due to the capacity of the receiving vessel, the ability to automate the monitoring of the gas increase and the delay due to initial pressure build up. Furthermore, it requires a measurement setup for each trial discouraging parallel experiments which are useful in assuring otherwise identical conditions.

The fermentation processes induced by kefir grains cause flocculation to occur, which is the process of the constituents of a solution aggregating [6] and forming a suspension which disintegrates easily with agitation. As the milk flocculates, the spacing between proteins will reduce which in turn will reduce the mean free path for diffusion. As seen in Equation 1, the magnetic resonance signal intensity for a given spin echo is increased as the diffusion coefficient or field gradient is reduced or $\mathrm{T}_{2}$ is increased. Since floculation will have an effect on the mean free diffusion path, this relationship shows that as the amount of flocculation increases, the brightness of the images collected using MRI should also increase, suggesting MRI will be a sensitive measure of fermentation. The amplitude of the Nth echo $A\left(N T_{E}\right)$ for an echo time of $T_{E}$, is given by

$$
A\left(N T_{E}\right)=M_{x y}(0) \cdot e^{-N T_{E} / T_{2}} \cdot e^{\frac{-D \gamma^{2} G^{2} N T_{E}^{3}}{12}},
$$

where $D$ is the self diffusion coefficient of water in a sample with spin-spin relaxation time constant $T_{2}$ with a magnetization at time zero of $M_{X Y}(0), \gamma$ is the proton gyromagnetic ratio and $\mathrm{G}$ is the gradient strength [14]. Whilst there is a possibility that there is also an inherent change in the value of $T_{2}$ which could also drive a change in signal intensity, the authors believe that the flocculation driven change in viscosity and diffusion will be the dominant effect. Since this echo amplitude is observed through imaging sequences, throughout the remainder of the manuscript, echo amplitude is expressed in terms of the grey-scale value of the resulting image pixel. 


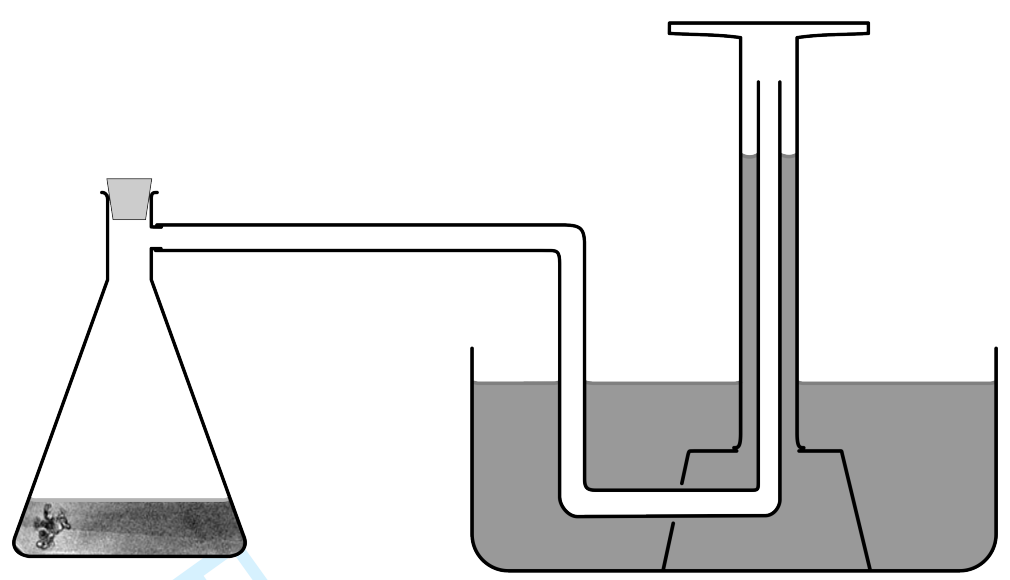

FIGURE 2 Schematic of the experimental setup used to correlate the production of gas with the change in MR signal intensity.

\section{2 | EXPERIMENTAL METHODS}

In this study, two experiments are undertaken to determine the effect of grain size on the fermentation process. The first is to validate the proposed MRI methods against traditional gas measurements. In the second experiment, the effect of different grain sizes on the fermentation process is also assessed using MRI. A whole body clinical MRI scanner (1.5T Magnetom Avanto, Siemens, Germany) is used for both of these experiments.

\section{1 | Validation of Magnetic Resonance Method}

In order to confirm that contrast of MRI images is a suitable measure of the fermentation of kefir milk, a traditional gas capture setup as shown in Figure 2 is produced using MRI compatible materials. Kefir grains weighing a total of ( $30 \pm 1) \mathrm{g}$ are placed inside a conical flask with $200 \mathrm{ml}$ of semi-skimmed milk. The conical flask is sealed and a gas collection tube attached to the side arm. The other end of the tube is placed in a water filled upturned measuring cylinder, with its open end immersed in a large water filled beaker.

This entire setup is placed on the MRI patient couch which is controllable from the console software. A four element flexible body coil is wrapped perpendicularly to the surface of the bed around the beaker for gas measurements whilst four elements of the built-in spine coil are used to image the fermenting milk, given its proximity. Weighted coil element signals are combined in k-space automatically by the scanner software to output a magnitude image without acceleration.

An MRI scan card automatically images the kefir once every hour, then moves the patient couch and images the volume of fluid remaining in the gas cylinder. This process is repeated for between 12 and 18 hours starting with a gas measurement.

An in-built Turbo Spin Echo (TSE) sequence with the following parameters is used to sagitally image the fermenting milk: Echo Time $=296 \mathrm{~ms}, \mathrm{~T}_{\mathrm{E}}$ eff of $2.6 \mathrm{~s}$ (optimized for the value of $\mathrm{T}_{2}{ }^{\text {eff }}$ according to [15]), repetition time of $2530 \mathrm{~ms}, 3$ averages, 20 slices, a flip angle of $150^{\circ}$ and an isotropic matrix size of $512 \times 512$ giving a spatial resolution of $(460 \times 460) \mu m$. The same sequence is used to image the gas set up over a single slice.

The central slice of the sample tubes from the kefir images was selected for image processing and is kept constant 
for all subsequent measurements. Image processing is undertaken using MATLAB (2017a, MathWorks, MA, USA) routines, written for this purpose. The user inputs the co-ordinates of the sample region of interest, thresholding is then performed to disregard low signal areas from the grains and gas bubbles as these otherwise act to reduce the increase in signal driven by flocculation. The signal intensity for the active region is then averaged (thus negating the effect of the variable number of thresholded voxels) and this value is plotted against time.

The measuring cylinder images are opened in ImageJ [16] to allow the the height of water in $\mathrm{mm}$ to be measured using a line profile. The spatial scale of the image was first be calibrated using the known height of the measuring cylinder. This process is repeated for all gas measurements. The length is multiplied by the cross sectional area of the cylinder and scaled to give the volume of gas in $\mathrm{ml}$.

To determine the relationship between gas production and MR signal intensity, the two resulting values are plotted against each other.

\subsection{Effect of Grain Size on Reaction Rate}

Five different kefir grain sizes with approximately equal mass are simultaneously imaged with MRI. The grains tested varied in size, initially being between $(2.9 \mathrm{~mm}$ and $13.5 \mathrm{~mm}) \pm 0.2 \mathrm{~mm}$ and after one fermentation growing to be between $3.9 \mathrm{~mm}$ and $14.8 \mathrm{~mm}$, as measured by ImageJ [16] from the MRI images. The Image J [16] measurements were performed on multiple grains where possible along the same axis due to the grains not being perfect spheres. The two largest of the five sizes are found as naturally occurring grains whilst the smaller three required breaking up larger grains. Whilst breaking the grains allows exploration of a wider range of grain sizes, given that the bacteria responsible for much of the fermentation process are supposedly found only on the outside, the overall active surface area is not increased to the extent it would be if the grains were naturally the same size.

A sufficient number of kefir grains are found or broken up to yield $(1 \pm 0.05) \mathrm{g}$ in mass. Each of the grain sizes are then placed into $50 \mathrm{ml}$ plastic centrifuge tubes along with $35 \mathrm{ml}$ of milk which are then sealed. These tubes are then placed in the MRI scanner, along with a tube of copper sulphate solution as a contrast reference. Images are collected using the same sequence as previously described but with images acquired every 15 minutes throughout the duration of the experiment and without the gas measurements.

A single slice is selected and kept constant for each image set collected. The same processing as used previously, is employed in MATLAB (2017a, MathWorks, MA, USA) but the averaged signal is now plotted against time.

The next day, prior to scanning, the kefir grains are removed from the kefirian milk, washed with tap water, placed into clean tubes with $25 \mathrm{ml}$ fresh milk and the imaging repeated. The change in the volume of milk is intended to allow exploration of the effect of milk volume to grain ratio on the rate of reaction.

As is expected from a biological process, the central region of the sigmoidal evolution in fermentation and hence in the signal intensity is approximately linear. This region is isolated and fitted with the inbuilt MATLAB (2017a, MathWorks, MA, USA) routine polyfit with a polynomial power of 1 providing a linear fit. The maximum and minimum possible slopes are estimated by skewing the data about the center value by the error value. The values of the slope of these fits, with error bars from the maximum and minimum fits are then plotted as a measure of the rate constant 


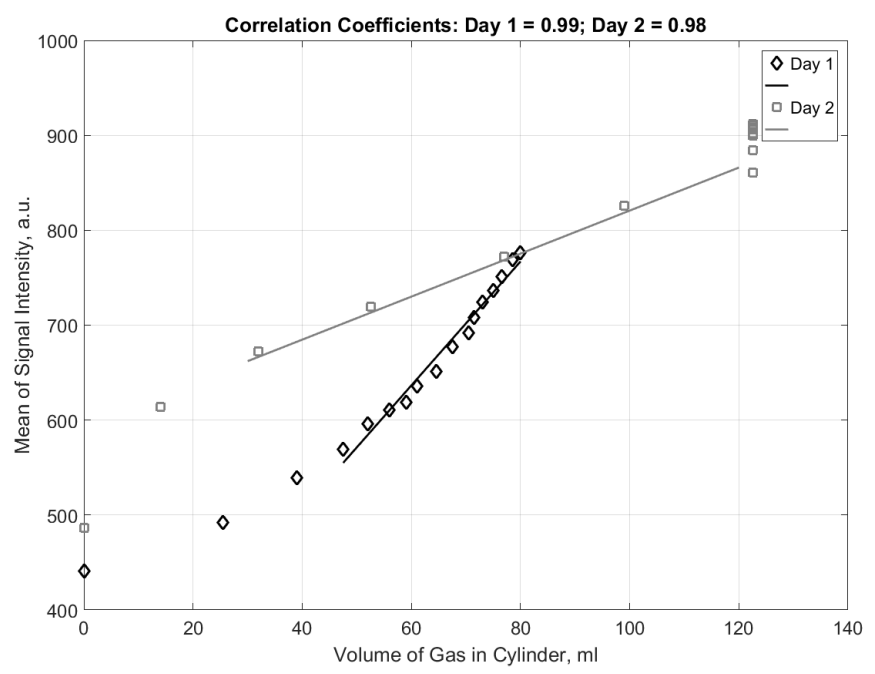

FIGURE 3 Plot of the averaged signal intensity as a function of the volume of gas produced during fermentation. The two experimental days are shown using Black Diamonds and Grey Squares respectively. The fits are for the portion of the curves after initial gas pressure has been established to displace the water in the measurement apparatus. The correlation coefficients for these linear regions are shown and verify that this portion is linear to a $95 \%$ confidence level.

\section{3 | RESULTS}

\section{1 | Validation of Magnetic Resonance Method}

The volume of gas produced as measured with ImageJ [16] and the averaged signal intensity after the processing previously described has been undertaken are plotted in Figure 3. It can be seen that the MR signal (the progression of which can be seen in Figure 4) responds almost immediately to flocculation changes, whilst the volume of gas requires an initial pressure to displace the water in the first experiment. In the second experiment, the gas tube is better purged and the gas data better follows the MR signal. The data which follow in two experiments are linearly fitted and found to have different slopes. The correlation coefficients are however similar at 0.99 and 0.98 verifying that the mean grey-scale value is proportional to the volume of gas produced during fermentation.

\subsection{Effect of Grain Size on Reaction Rate}

Figure 5 shows the evolution of the signal intensity for the different sized kefir grains on two days with different volumes of milk. Day one is with $35 \mathrm{ml}$ of fresh milk and Day two is with $25 \mathrm{ml}$ of fresh milk. Each grain size is displayed in a different color whilst the different marker shapes show the different milk volumes (Crosses are $35 \mathrm{ml}$, Circles are $25 \mathrm{ml}$ ).

For each sample, results from both experiments tend to the same point with each also appearing to have similar slopes once the fermentation has fully started. Overall it appears that as the grain size decreases the rate of reaction increases as does the overall fermentation level. In order to evaluate the reaction rates, the linear portion of the fermentation process is analyzed as detailed in the experimental methods section and is shown in Figure 6a, whilst the maximum signal intensity is evaluated in Figure $6 \mathrm{~b}$. 


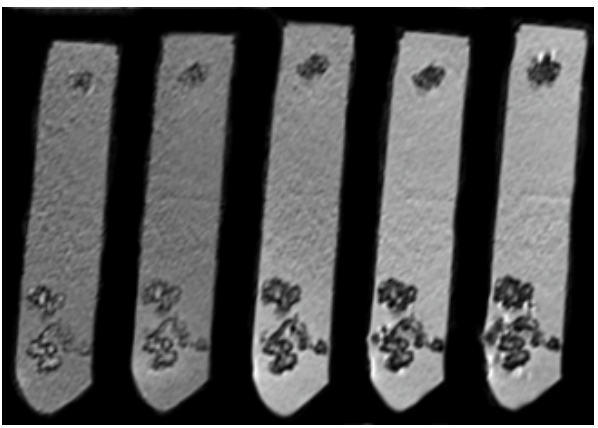

FIGURE 4 The time-course of the evolution of the MR signal for normal grains. Here the increase in grey scale value from left to right represents the change in the signal intensity from low (black) to high (white). Bulk fermentation of the milk can be seen as a change from low to high signal intensity in the fluid volume.

A simple model can be used to predict the rate of the reaction as a function of grain size. First it is assumed that the mass of the grains is proportional to the cube of their size $R^{3}$. As each tube contains approximately the same total mass, the number of grains in each tube must scale as $R^{-3}$. The next assumption is that the surface area of each grain is proportional to the square of the radius, $R^{2}$, leading to the observation that the total surface area of kefir within each tube is inversely proportional to $R$. Finally, if it is assumed that the rate of signal change, which is due to the reaction taking place at the surface, is proportional to the surface area, Therefore a prediction can be made that rate should scale with $R^{-1}$. Two curves of this form are fitted to the data and plotted on Figure 6a, showing good agreement.

\section{4 | DISCUSSION}

\section{1 | Validation of Magnetic Resonance Method}

The linear relationship between the volume of gas produced and the average pixel intensity means that the use of MRI is a suitable measure of the flocculation and hence fermentation process. The lag between the signal intensity changes and the volume of gas produced in the first experiment is due to the initial pressure which must be achieved to displace the water from the gas tube whilst the latter points forming a vertical line are as a result of producing more gas than could be contained in the measuring cylinder whilst the MRI continues to be sensitive. In the second day's experiment the gas line was fully cleared of water before the experiment resulting in better agreement between the data.

An important finding from this experiment was the difference between the slopes of reaction rates for the two days. It was discovered that this was due to the automatic change of the grey-scale value in the MRI imaging software which was unreliably scaling the data. In order to prevent this in future measurements a sample tube containing two concentrations of copper sulphate solution was included in subsequent scans to use as a maximum signal reference. The raw signal from this sample should remain constant hence dividing all measurements by the value of this sample corrects for image contrast drift.

\subsection{Effect of Grain Size on Reaction Rate}

There are a number of features which can be seen on the plot in figure 5 . The first is that the maximum value reached by each grain size is similar. Furthermore, the smaller the grain, the higher the ultimate signal intensity. The authors 


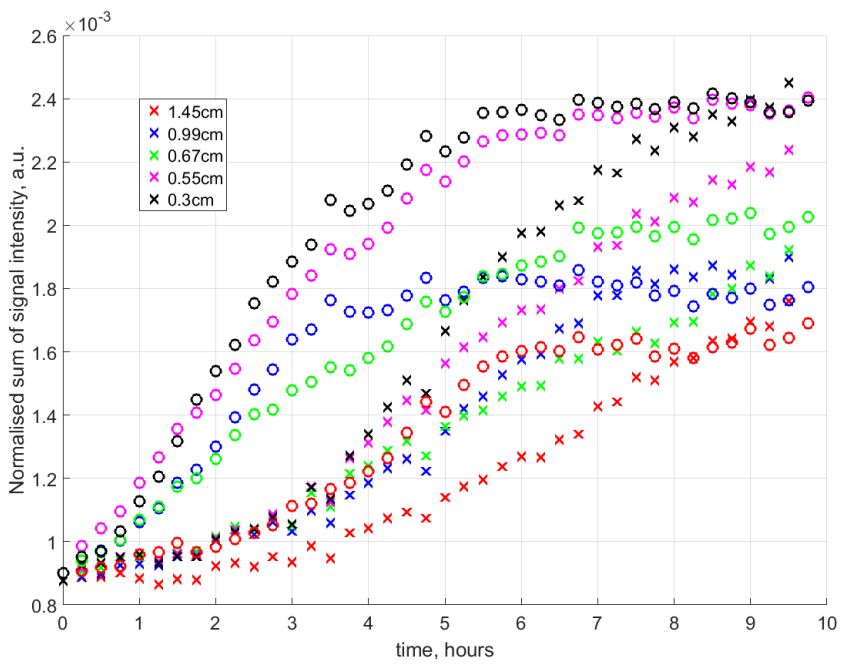

FIGURE 5 Plot to show the variation in the mean signal intensity as a function of time for two experimental runs with five grain sizes. The first run is in $35 \mathrm{ml}$ milk and is shown with crosses, with each grain size encoded by a color. The second run is in $25 \mathrm{ml}$ of milk and is shown with circles using the same color coding. The legend details the grain size for each color. Note the similar reaction rates and end points for a given grain size. Similarly, the smaller the grain size, the higher the final signal intensity.

believe that this is due to a decrease in the availability of the bacteria to the bulk milk distant from larger grains, as local flocculation decreases diffusive migration. This is supported by the data in Figure $6 \mathrm{~b}$ displaying the maximum signal intensities achieved. The smaller grains, providing less local restriction to diffusion lead to a higher level of fermentation of the bulk, whilst the larger grains tend to result in lower levels of flocculation overall. The variation in the reaction rates of the samples is best seen in Figure 6a. Accounting for the change in grain size as they undergo growth, results in the points laying in two linear regions, with the reaction rate for each grain lying within error of the second iteration. This suggests that the milk to grain ratio is not as important as previously reported in the literature. Further experimentation is however needed to verify this. The change in these regions happens where the grains are produced by breaking up larger grains instead of with naturally occurring sizes. The authors believe that the action of breaking them up exposes the inner layer which is not as biologically active from a fermentation perspective. This results in a drop off of their overall biological action which is slowly re-colonised by the bacteria. The smaller the resulting grain however, the higher the ratio of the bacterial surface to the inactive extracellular matrix and thus the quicker they are fully re-colonised. This result is of key importance to the production of kefir. It demonstrates that breaking up larger grains into smaller grains can be beneficial to both the rate of production and also the final kefirian milk properties. However it is important that the grains are well broken up to maximize this effect. Furthermore, the properties of the final product can be varied by arresting the process at different times along the fermentation.

\section{3 | Fermentation Variations}

For the majority of grains tested, fermentation was seen as the bulk flocculation of the milk. However for a small number of the initial grains, ranging in size between $10-20 \mathrm{~mm}$, a different behaviour was observed. Instead of driving the 

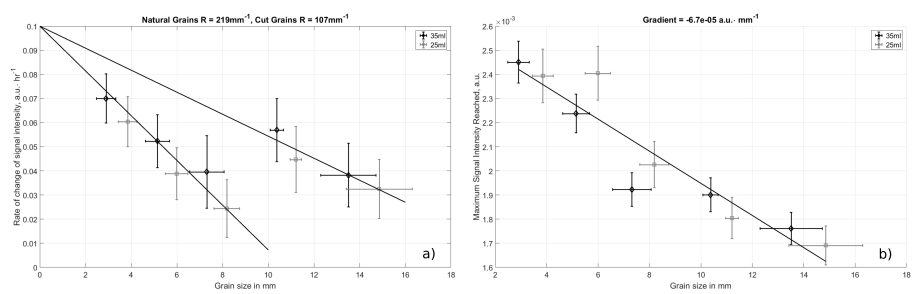

FIGURE 6 a) Plot of the rate constants of fermentation as a function of grain size. Initial grains are represented by black diamonds, the grains after 24 hours of growth are represented by grey squares. The X errors are estimated from repeated measurements of grain size in ImageJ[16] whilst the y errors are the standard error from the averaging of the MRI voxel intensities. The appearance of two linear regions occurs where the grains are broken up instead of forming naturally. The authors believe this is due to the exposure of the less active extracellular matrix at the core of the grains thus not increasing the active surface area as much as the overall surface area is increased. b) Plot of the ultimate signal intensity reached during the fermentation process as a function of grain size. The two colors represent consecutive fermentations with black diamonds for the initial grains and grey squares for the grains after a fermentation cycle. The spacing on the $x$ axis between these is due to their growth. Unlike the data for flocculation rate as shown in a), there is no obvious difference between the naturally occurring grains and those which have been split into smaller pieces.

complete flocculation of the bulk milk, the kefir grain produces a spherical shell of densely flocculated material which appears impervious to further biological action. A photograph of the resulting grain and a corresponding MRI image are showing in figure 7. The authors are not aware of any other reports of this in the literature or anecdotally through home producers. At this time the reasoning behind this is unclear and further investigation is needed with controlled milk environments to reproduce the results. The authors believe that this could be a stress response to unfavorable $\mathrm{pH}$ conditions of previous fermentations leading to a permanent change but have no measurements to substantiate this prediction. An alternative explanation could be due to changes in $\mathrm{T}_{2}$ or diffusion driven by locally rapid protein precipitation for the smaller grain size although such behaviour is not repeatably observed. Further experiments are necessary to determine the exact mechanism underlying these results. 

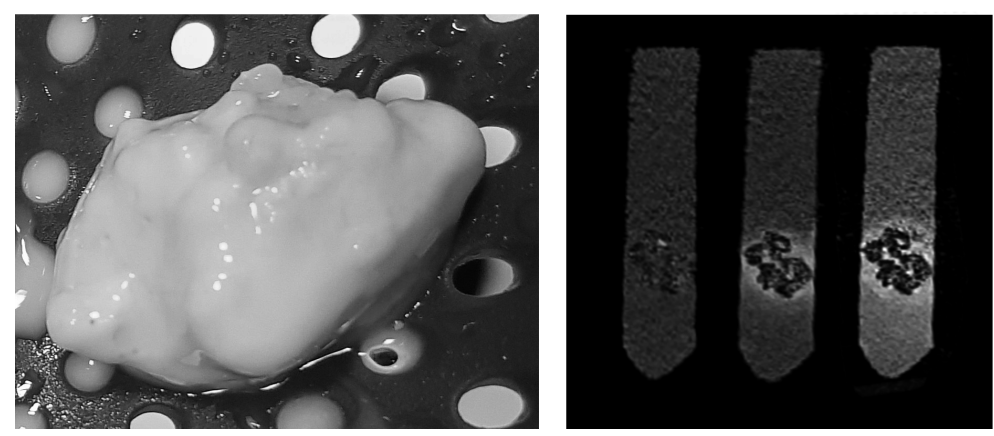

FIGURE 7 Kefir grain with different behaviour. Left hand side is a photograph of a grain. The right hand side image is the time-course of the evolution of the MR signal in the milk sample containing the same grain. Notice the rapid onset of a bright perimeter to the grain in the right image which is not present on Figure 4, where the bulk material is instead affected.

\section{5 | CONCLUSION}

It has been demonstrated that Magnetic Resonance Imaging is a useful tool in monitoring the fermentation of milk through the action of kefir grains by comparing the production of gas with the mean MR signal intensity over an image region of interest. This has then been used to monitor the fermentation rate and extent in multiple grain sizes simultaneously thus eliminating inter-experiment variation due to external factors such as temperature. It was found that breaking larger kefir grains into smaller pieces yields a faster reaction rate (0.07 a.u. per hour vs 0.038 a.u. per hour) so long as they are sufficiently small since intermediate values have a reduced reaction rate (as low as 0.025 a.u. per hour) owing to exposure of the inactive extracellular matrix. This may offer an improvement in the ability to control the resulting kefirian milk product whilst maximizing the speed of production which may be of benefit to home producers and industrial producers alike. Future work should investigate how best to precondition the kefir grains in order to improve consistency and to better understand the localised fermentation behaviour displayed by a small number of sample grains.

\section{ACKNOWLEDGEMENTS}

AS thanks the Nottingham Trent University Physics Undergraduate Research Scholarship (PURS) program for providing support. The authors thank Dr Benjamin Dickins, Dr Neil Williams and Dr Kirsty Hunter for their assistance in the conceptual development of this experiment.

\section{CONFLICT OF INTEREST}

The authors do not have any conflicts of interest.

\section{REFERENCES}

[1] Pintado ME, Da Silva JAL, Fernandes PB, Malcata FX, Hogg TA. Microbiological and rheological studies on Portuguese kefir grains. International Journal of Food Science and Technology 1996 feb;31(1):15-26. http://doi.wiley.com/10. $1111 / \mathrm{j} .1365-2621.1996 .16-316 . x$. 
[2] Garrote GL, Abraham AG, De Antoni GL. Characteristics of kefir prepared with different grain milk ratios. Journal of Dairy Research 1998;65(1):149-154.

[3] Nielsen B, Gürakan GC, Ünlü G. Kefir: A Multifaceted Fermented Dairy Product. Probiotics and Antimicrobial Proteins 2014 dec;6(3-4):123-135. http://link. springer. com/10. 1007/s12602-014-9168-0.

[4] Beshkova DM, Simova ED, Simov ZI, Frengova GI, Spasov ZN. Pure cultures for making kefir. Food Microbiology 2002;19(5):537-544. http://www.idealibrary.com.

[5] Marshall VM, Cole WM. Methods for making kefir and fermented milks based on kefir. Journal of Dairy Research 1985 aug;52(03):451. http://www . journals . cambridge.org/abstract/S0022029900024353.

[6] Domingues L, Vicente AA, Lima N, Teixeira JA. Applications of yeast flocculation in biotechnological processes. Biotechnology and Bioprocess Engineering 2000 aug;5(4):288-305. http: //link. springer. com/10.1007/BF02942185.

[7] Bottazzi V, Bianchi F. A Note on Scanning Electron Microscopy of Micro-organisms associated with the Kefir Granule. Journal of Applied Bacteriology 1980 apr;48(2):265-268. http: //doi . wiley . com/10.1111/j .1365-2672 .1980.tb01225. $\mathrm{x}$.

[8] Yaman H, Elmali M, Karadagoglu G, Cetinkaya A. Observations of Kefir Grains and Their Structure From Different Geographical Regions: Turkey and Germany; 2006.

[9] Osimani A, Garofalo C, Aquilanti L, Milanovic V, Scibè C, Sarra S, et al. Il potenziale biologico del kefir: studi preliminari del microbiota di grani di kefir italiani.The biological potential of kefir: preliminary studies of the microbiota of italian kefir grains. Industrie Alimentari 2014 06;53(547):12-20.

[10] Marshall VM. Starter cultures for milk fermentation and their characteristics. International Journal of Dairy Technology 1993 may;46(2):52-53. http://doi .wiley.com/10.1111/j.1471-0307.1993.tb00860.x.

[11] Garrote GL, Abraham AG, De Antoni GL. Chemical and microbiological characterisation of kefir grains. The Journal of dairy research 2001 nov;68(4):639-52. http://www.ncbi.nlm.nih.gov/pubmed/11928960.

[12] Plessas S, Pherson L, Bekatorou A, Nigam P, Koutinas AA. Bread making using kefir grains as baker's yeast. Food Chemistry 2005 dec;93(4):585-589. https : / /www . sciencedirect .com/science/article/pii/s0308814604007782.

[13] Zaki El-Din M, El-Shazly K. Evaluation of a Method of Measuring Fermentation Rates and Net Growth of Rumen Microorganisms; 1969.

[14] Blumich B. NMR imaging of materials. Oxford University Press; 2003.

[15] Morris RH, Bencsik M, Nestle N, Galvosas P, Fairhurst D, Vangala A, et al. Robust spatially resolved pressure measurements using MRI with novel buoyant advection-free preparations of stable microbubbles in polysaccharide gels. Journal of Magnetic Resonance 2008 aug;193(2):159-167. https://www. sciencedirect.com/science/article/pii/ S1090780708001195.

[16] Schneider CA, Rasband WS, Eliceiri KW. NIH Image to ImageJ: 25 years of Image Analysis. Nature methods 2012 jul;9(7):671-675. http://www.ncbi.nlm.nih.gov/pmc/articles/PMC5554542/. 